\title{
¿Combate a la corrupción o cambio político? La construcción político-judicial contra el Partido dos Trabalhadores en Brasil
}

\author{
Josep Pont Vidal \\ UNIVERSIDADE FEDERAL DO PARÁ (UFPA), BELÉM, BRASIL \\ josevidal@ufpa.br
}

Resumen: La decisión judicial de encarcelar al ex presidente Luiz Inácio Lula da Silva es la culminación de un largo proceso político y judicial iniciado y fundamentado en el conjunto de investigaciones conocidas como la Operação Lava Jato (Operación túnel de lavado). El detonante ha sido un complejo proceso político que culminó con el empeachment de la Presidente Dilma Rouseff y el nombramiento de su vice-Presidente Michel Temer como Presidente del país en el año 2016. A partir de una hipótesis sustantiva principal sobre la predeterminación del código "no poder", el objetivo de este trabajo es exponer este proceso en el plano teórico-conceptual el análisis de las comunicaciones en los sistemas político-administrativo y jurídico, y su construcción dirigida contra el Partido dos Trabalhadores (PT). Destaca el papel de los medios de comunicación de masas en la construcción de este proceso. El análisis teórico de sistemas autorreferenciales se ilustra documentos procedentes de los procesos de empeachment y de corrupción contra Lula. Como conclusiones formulamos unas hipótesis sobre si este proceso jurídico realmente ha sido un modelo a seguir de combate a la corrupción.

Palabra clave: sistema político-administrativo, comunicaciones, corrupción, códigos, Brasil

Abstract: The judicial decision to imprison former President Luiz Inácio Lula da Silva is the culmination of a long political and judicial process initiated and based on the set of investigations known as "Operação Lava Jato" (Operation car wash). This operation was boosted by a complex political process that also generated the impeachment of Brazilian President Dilma Rouseff and the designation of her Vice-President Michel Temer as President of the country in 2016. On the basis of a main substantive hypothesis, about the predetermination of the "can not" code, this paper aims to expose this process, in the theoretical-conceptual plane, the analysis of communications in the Brazilian political-administrative and legal systems, and its construction directed against the Workers Party (PT). It emphasizes the role of the mass media in this process construction. The theoretical analysis of self-referential systems, is based on documents from the impeachment and corruption process against Lula. As conclusion, some hypotheses are proposed as to whether this legal process has really been a model for fighting corruption.

Key words: political-administrative system, communications, corruption, codes, elections, Brazil 


\section{Introducción}

lingreso en prisión del ex presidente Luiz Inácio Lula da Silva como consecuencia de la decisión inapelable del Juez Sérgio Moro (titular de la 13 Vara Criminal $\checkmark$ Federal de Curitiba) (Justiça Federal. Sentença, 2018) y del $8^{\circ}$ Tribunal Regional Federal de la 4a región (TRF-4) es la culminación de un largo proceso escenificado por la conjunción de los sistemas político-administrativo y judicial con el apoyo incondicional de los medios de comunicación de masas, cuya construcción se inicia con aspectos de su salud (Fortuna, et al., 2012). La solicitud de un habeas corpus al Supremo Tribunal Federal (STF) fue rechazada por seis votos a cinco. Durante todo el proceso se han puesto de manifiesto una serie de parcialidades del sistema jurídico ante la falta de pruebas concluyentes. Este hecho marca un precedente histórico en el sistema judiciario y político-administrativo cuyos antecedentes se encuentran en el primer mandado del presidente Lula (2003-2011) durante el año 2005 en que fueron denunciadas prácticas irregulares del "mensalão" (compra de votos de diputados del Congreso Nacional de la oposición con el objetivo de aprobar leyes y propuestas por el ejecutivo). Se inicia en la conocida Operação Lava Jato (Operación túnel de lavado) iniciada el 17 de marzo del año 2014 (y que entretanto ya ha tenido 50 fases operativas hasta abril de 2018) contra la corrupción en todo el país cuyo detonante ha sido un complejo proceso político que culminó con el empeachment de la Presidente Dilma Rousseff (2011-2016)(Senado Federal, 2016; 2016a 2016b) y el nombramiento de su vice-Presidente Michel Temer como Presidente del país en el año 2016.

Las reacciones nacionales e internacionales no se han hecho esperar, abarcando desde las declaraciones de condena de expresidentes (Massimo d'Alema, Romano Prodi), Barack Obama que en su día lo definió como "el político más popular de la Tierra" al preso más famoso de la región, hasta el apoyo del Premio Nobel Adolfo Pérez Esquivel. Contrastan reacciones y acciones violentas apoyando esta decisión. Entretanto el país se encuentra dividido entre "Lula fuera de la prisión" y la "demanda de militares al poder". Diversas publicaciones independientes y académicas han manifestado los logros sociales durante los gobiernos del PT (De Paula, 2011; Aguiar, 2015, Zahluth, 2017) con un alza de un 3,3 por ciento en la bolsa de São Paulo. El jurista Dalmo Dallari sintetiza este proceso con una interpretación autorreferencial: "el sistema político se ha autodestruido y la democracia fue junto" (Vermelho, 19 diciembre, 2017), mientras que para Tarso Genro en Brasil se ha instaurado "el Estado-espectáculo".

Estos hechos de índole política y jurídica, han llevado a una división de la sociedad brasileña que cobra día a día cada nuevas manifestaciones (ataque armado a la caravana electoral de Lula, incitación al odio político, manifestaciones contrarias en todo el país etc.) La complejidad del fenómeno induce a la formulación de una 
serie de cuestiones: ¿Se ha tratado de un cambio de poder político construido por el sistema político-administrativo y el judicial?, ¿En qué se diferencia la acusación de corrupción del expresidente Lula de otros presidentes con casos incluso más graves que fueron acusados pero no enjuiciados? ¿Son conclusivas las pruebas contra Lula? ¿Cuál ha sido el papel de los medios de comunicación de masas en esta situación de construcción política de la realidad? Son muchas preguntas.

En el campo la Ciencia política y la Administración pública, hoy por hoy no existen fundamentos teóricos indiscutibles para dar respuestas a la complejidad de este tipo de fenómenos socio-políticos. Los conceptos centrados solo en "el hombre" han mostrado su saturación, arbitrariedad metodológica y limitación explicativa para la descripción en el plano macro. Sucede de forma similar en el foco del actor que limita una percepción más amplia del fenómeno. Conceptos antes indiscutibles de estas disciplinas (poder, estado) como conceptos básicos solo pueden explicar aspectos parciales del todo. La idea de Estado sigue siendo una categoría indeterminada y analíticamente poco útil además de estar sujeta al análisis procedente de la tradición y los prejuicios que recaen en ella. Por otro lado, la noción de gobierno sufre de la debilidad opuesta, aunque más precisa en términos de lo institucional y organizacional, deja de tener capacidad auto-explicativa, al tratarse de una categoría orientada a la acción. Esta situación epistemológica en la Ciencia política y la Sociología abre la puerta a experimentar a la utilización de conceptos y categorías vinculados sobre la construcción de la política de la realidad que permitan un abordaje desde la complejidad. En este sentido, la teoría sociológica y politológica de sistemas autorreferenciales de Niklas Luhmann y aportaciones posteriores neosistémicas, politológicas y sociológicas (Willke, 2016; 1997; Nassehi, 2008; Japp, 2008; Japp y Kusche, 2004; Farías y Ossandón, 2011; Kooimann, 2008) y pos-estructuralistas (Leydersdorff, 2015) constituyen un marco que permite la observación y análisis dinámico de las comunicaciones y operaciones internas entre los sistemas que forman la sociedad.

En base a estas observaciones previas, este trabajo está organizado del siguiente modo. En primer lugar, en forma de contextualización describimos el escenario el proceso judicial contra el ex presidente Lula. Exponemos de forma cronológica el proceso jurídico por las que ha sido acusado. Acto seguido presentamos la propuesta de idea de la construcción política de la realidad política a partir de la teoría de sistemas autorreferenciales de Luhmann y aportaciones posteriores neosistémicas. Por último describimos los códigos, operaciones y comunicaciones básicas entre el sistema político-administrativo y el sistema judicial. Al abarcar diversos subsistemas nos limitaremos a una descripción sucinta del fenómeno de las operaciones directamente relacionadas con la corrupción, los códigos emitidos por los subsistemas en los que el sistema político-administrativo actúa como principal conductor y de dirección. 


\section{Hipótesis y metodología}

Dada la multiplicidad de factores y variables que intervienen en este complejo fenómeno jurídico-político, requiere un abordaje que sobrepase lo la división clásica disciplinaria entre Ciencia Política, Sociología y Administración. Por ello, lo abordamos en la perspectiva de Sociología política (Rattingen, 2009; Böhnisch, 2006; Luhmann, 2014) cuyos "interfaces" y fronteras entre las disciplinas ya han sido identificados y tratados (Llera Ramo, Luhmann, Mayntz, Schmitter, Bourdieu) en una perspectiva interdisciplinaria. Las cuestiones formuladas permiten la formulación de unas hipótesis.

La hipótesis sustantiva que formulamos viene a ser que en la capacidad operativa del derecho ha recibido y operado comunicaciones en forma de códigos procedentes del sistema político-administrativo y del sistema jurídico, -en el que el código "legalilegal" ha sido sustituido por el "poder-no poder", prevaleciendo esta último de tal forma que han tenido como resultado unas decisiones fundamentadas en pruebas partidistas de los casos procesados. Con este proceso, sin embargo, el PT puede aparecer como el partido de la oposición, con lo que puede "hacer valer el poder de los no poderosos" (Luhmann, 1993:163), con lo que se generaría una "inestabilidad en el sistema". La fórmula ha sido el desprestigio del PT al encuadrarlo como "el partido corrupto". Hemos elaborado dos variables relacionadas con el papel del sistema jurídico y del político administrativo relacionados con el empeachment de Dilma Rousseff y reclusión del expresidente Lula:

- El análisis de los códigos y las comunicaciones en forma de las sentencias emitidas por el sistema jurídico respecto estos hechos políticos;

- El análisis de las comunicaciones en forma de acusaciones emitidas por el sistema político-administrativo respecto a la construcción de corrupción de los referidos expresidentes.

En el plano de la comunicación política, las comunicaciones han estado dirigidas a una necesidad de sustitución de los partidos y actores en el poder mediante la presentación pública en los medios de comunicación de masas del Partido dos Trabalhadores como el "único partido corrupto" y como "partido sistémico corrupto". Con estos juicios se ha impedir la candidatura del expresidente Lula que representa unas expectativas posibles de un nuevo triunfo en las elecciones generales de otoño de 2018, y a su vez desgastar públicamente el PT en los planos social y político.

$\mathrm{Al}$ existir pocas posibilidades de que la derrota del PT pudiese tener lugar por medio de elecciones democráticas, los partidos en el poder han recurrido a dos estrategias: primero, mediante construcciones jurídicas de escaso peso; segundo, a los medios de comunicación de masas con el objetivo de crear un clima de 
inseguridad y de decisión pública favorable a la necesidad del mantenimiento de un orden de gobernanza política más eficaz y jerárquico (Börzel y Risse, 2010; Mayntz, 2009, 2005).

En el plano metodológico el trabajo se limita a la ilustración de la hipótesis sustantiva y variables que se describen y analizan en el plano teórico-conceptual, y en las que los datos e informaciones solo sirven de forma ilustrativa. Los datos e informaciones que se acompañan no pretenden mostrar una observación fundamentada en deducciones analógicas, sino que se limitan a la simple ilustración de los conceptos y comunicaciones entre los referidos sistémicas. Las informaciones proceden de fuentes secundarias publicadas entre los años 2003-2018, a partir de los siguientes criterios y fuentes:

- Publicaciones de las instituciones gubernamentales federales y estaduales, Diario Oficial da União (DOU), Senado Federal, Ministério Público Federal (Ministerio Fiscal en España), Supremo Tribunal Federal (STF), Superior de Tribunal Justiça, Congreso Nacional, Policía Federal (Ministério Extraordinário da Segurança Pública), Banco de Datos Eleitorais;

- Documentos producidos por los gabinetes de los abogados de Lula (Martins, Martins Advogados) y de Dilma Rousseff (Engelber y Mattos Advogados);

- Informaciones producidas por los principales medios de comunicación (TV y escritos);

- Artículos publicados procedentes de centros de investigación y documentos de los partidos políticos directamente vinculados con los procesos.

\section{El escenario}

El triunfo electoral del Luiz Inacio Lula da Silva en el año 2003, marco un nuevo periodo en el Brasil dando inicio un proceso de reformas sociales e institucionales sin precedentes en el país. Algunas de estas iniciadas tímidamente por su predecesor el sociólogo Fernando Henrique Cardoso (1995-2003). El triunfo significó la conquista del poder de un partido surgido del movimiento sindical (Central Única de Trabajadores) en el que participaron diversos sectores de la sociedad: movimientos cristianos de base, Movimento Trabalhadores Rurais sem Terra (MST), Movimento Trabalhadores sem Teto (MTST) entre otros movimientos y grupos. Su candidatura estivo apoyada por diversos partidos minoritarios que se integrarían en el primer gobierno. En el primer turno obtuvo el 46,4 por ciento de votos y en el segundo el 61,3 por ciento (equivalente a un total de 52.772.475 millones de votos) (Tribunal Superior Eleitoral, 2003). Su triunfo fue visto en la prensa internacional como un fenómeno inédito: la prensa de Israel lo calificó como “El profeta del diálogo” (Haaretz, BBC, 12, marzo, 2010), Le 
Monde lo declaró como "hombre del año en el 2009" (Le Monde y El País, 2009), el Financial Times "Lula es una de las 50 personas que cambiaran la década" (Financial Times, 29 diciembre 2009) y la revista Forbes como "The World's Most Powerful People". En el año 2008 Brasil conquisto el grado de investment grade (grado de confianza en la inversión) por la agencia de riego americana Standart \& Poor's. Sin embargo, las reacciones en el interior fueron muy diferentes, proviniendo las críticas tanto de los sectores conservadores, al calificarlo de "conducir al país al comunismo", como de los sectores más radicales, del interior del su partido, como de otros sectores de la sociedad civil. Desde el marxismo radical se extendió la crítica: "el gobierno Lula fue capaz de reponer y consolidar el neoliberalismo como programa político del bloque en el poder; atrayendo para a hegemonía del capital financiero el apoyo activo de las principales organizaciones del mundo del trabajo"' . Con la Carta ao Povo Brasileiro (2002) fueron cuestionados los fundamentos de su programa y de su giro socialdemócrata. Esta Carta, estaba destinada a crear confianza entre los empresarios brasileños y el mercado mundial, y se responsabilizó de mantener las metas y la inflación y la política macro-económica adoptadas por el anterior presidente, además de mantener el pagamento de la deuda externa con el FMI: "Premisa de esta transición será naturalmente o respecto a los contratos y obligaciones del país (...) Este es el mejor camino para que los contratos sean honrados y el país recupere a libertad de su política económica”.

Aprovechando la crisis interna del partido en el año 2003 miembros de PT disidentes de las políticas macro-económicas cercanas a la economía neoclásica y al consenso de Washington fueron expulsados del partido al no seguir indicaciones para la votación de la reforma de las jubilaciones. Esta situación juntamente con la publicación del escándalo de corrupción - "mensalão" - entre los años 2003 y 2006 contribuyo a que sectores radicales del marxismo emigraron al Partido Socialismo e Libertade (PSOL) hasta aquella fecha un pequeño y desconocido partido radical. En posteriores comicios municipales, estaduales y federales, los candidatos del PSOL se mantendrían como partido minoritario tanto por el número de votos como de representación de los movimientos sociales. El partido tendría destaque internacional a raíz del asesinato el 14 de marzo de 2018 de la activista y concejal de Rio de Janeiro Marielle Franco y su chófer Anderson Gomes. La concejal asesinada era defensora de los derechos humanos, activista antirracista, en defensa de los homosexuales y se oponía a la intervención militaren en Rio de Janeiro al denunciar grupos parapoliciales como responsables de la situación de violencia que viven las comunidades de la ciudad. El día 8 de abril era asesinado el líder comunitario Carlos Alexandre Pereira en Río de Janeiro.

1. Profesor David Maciel Universidade Federal de Goiás, en: https://www.ifch.unicamp.br/formulario_cemarx/selecao/2012/trabalhos/7355_Maciel_David.pdf 


\section{El proceso judicial contra el ex presidente Lula}

Para el Ministerio Público Federal, se trata del mayor caso de corrupción y de lavado de dinero ya descubierto en el Brasil, al estar vinculado con numerosos políticos, cargos municipales, administradores, gerentes de empresas (Petrobras, Odebrecht, JBS, Puerto de Santos, entre otras). El 12 de julio del 2017 se condenó el ex-presidente Lula a nueve años y seis meses de prisión.

Es la primera vez en la historia del Brasil en que se condenó criminalmente a un ex-presidente da República. Los tres ministros de la $8^{\text {a }}$ Turma del TRE-4 votaron por mantener la condenación y ampliar la pena de prisión de Lula en relación a la sentencia del Juez Federal Sérgio Moro. La decisión unánime redujo la única posibilidad de un recurso de Lula en el TRF-4 (los llamados embargos de declaración), que pueden ser juzgados por los mismos magistrados, pero sin poder para revertir la condena. Exponemos a continuación una síntesis argumentativa del proceso judicial ${ }^{2}$. El argumento de los jueces en sus votos fue la siguiente:

1) Lula recibió propina de la empresa contratista OAS en forma de un apartamento triplex en el municipio de Guarujá;

2) la propina resultó de un esquema de corrupción organizado en la empresa Petrobras;

3) el dinero procedió de una cuenta de la OAS destinada a financiar el PT como pago por el favor a la empresa en contratos con la Petrobras;

4) aunque no haya habido transferencia formal de contrato del apartamento para Lula, el apartamento fue reservado para él, lo que configura tentativa de ocultar el patrimonio (lavado de dinero);

5) aunque no exista una "acto de ofício" (escritura), en la forma de contrapartida a la empresa, solamente el haber aceptado la promesa de recibir ventajas indebidas mediante el poder de conceder el beneficio a la empresa se configura como un actor de corrupción;

6) los hechos investigados en la Operación Lava Jato revelan prácticas de compra de apoyo político de partidos idénticas a las del escándalo del "mensalão";

7) el juez Sérgio Moro, cuya imparcialidad es respondida por la defesa, era apto para juzgar el caso.

La defensa de Lula negó estas acusaciones con los argumentos: 
1) dice que él no es propietario del apartamento;

2) que no existen pruebas de que el dinero obtenido por la OAS en contratos con la Petrobras fuera utilizado en el apartamento;

3) que, de acuerdo con esta tesis, Moro, responsable por la conducción del Lava Jato, no podría haber juzgado el caso;

4) que el juez actuó de forma parcial;

5) que Lula es objetivo de persecución política.

Incluso después de la proclamación de veredicto, 1) Lula no será preso de inmediato; eventual prisión solo después del juicio del último recurso de la defesa al tribunal;2) la defesa puede recurrir al Superior Tribula de Justicia y al Superior Tribunal Federal para intentar revertir la condena; 3) el PT podrá registrar la candidatura de Lula como presidente; 4 ) la candidatura podrá ser mantenida mientras haya la posibilidad de recursos pendientes contra la condenación; 5) el Tribunal Superior Electoral decidirá si podrá ser inelegible.

Lula fue acusado pelo Ministerio Público de recibir propina de la empresa contratista OAS. La supuesta ventaja (por un valor de 2,2 millones $\mathrm{R} \$$ ), tendría procedido de una cuenta de propina destinada al PT a cambio de favores de la empresa en contratos con la Petrobras. Según el MP, la ventaja fue pagada en forma de reserva y reforma del apartamento cuya propiedad tendría sido ocultada. Una de las declaraciones principales que se fundamentó la acusación del Ministerio Público y la sentencia del Juez Moro y del ex-presidente de la OAS Léo Pinheiro, también condenado en el proceso.

\section{La construcción de la realidad en la óptica sistémica}

Hemos expuesto de forma cronológica unos hechos que pretender constituir una realidad en la construcción jurídica en la que la realidad del derecho es también una construcción social y en la que el sistema jurídico depende de un conjunto de creencias "compartido por las personas relevantes" como ha formulado Vilajosana (2010:4). En el caso presentado estas "personas relevantes" lo constituyen los actores políticos en el poder. En la Sociología construccionista la realidad se entiende como los fenómenos construidos socialmente, mientras que en la Ciencia Política se entiende como fenómenos políticos, en los que el "conocimiento" pasa a ser la información sobre las características de esos fenómenos. Las dos disciplinas científicas parten de supuestos diferentes sobre la construcción de la realidad del orden social, al privilegiar la interacción entre actores (Ciencia política) o la interacción entre sujetos y agentes (Sociología). Sin embargo, en un enfoque interdisciplinario ambas tienen 
como referencia inicial las hipótesis sobre la construcción de la realidad social de Alfred Schutz ([1932]1972) en su perspectiva de la fenomenología social y la importancia de la intersubjetividad ${ }^{3}$.

La perspectiva sistémica del construccionismo sistémico ("construccionismo operativo") de Niklas Luhmann, en la óptica de la sociología política (Luhmann, 2014, 1987, 1984) constituye un nexo entre los supuestos iniciales de ambas disciplinas al interesarse por las operaciones de los diversos subsistemas constituyentes del sistema social. En su presentación de la sociedad Luhmann (1984) prescinde de los supuestos lingüísticos y los sustituye por la comunicación entre los sistemas autorreferenciales. Estos pueden definirse como un tipo de sistema que produce sus propias operaciones, o en otras palabras que tiene la capacidad de producirse a sí mismos. Son sistemas que están clausurados en su operación, lo que les permite la autoorganización de que las estructuras y los elementos puedan formarse en su interior (autopoiesis) aunque cognitivamente abiertos. No vamos a extendernos más aquí sobre esta cuestión al sobrepasar los objetivos de este escrito y al disponer de una literatura especializada sobre el tema (Izuzquiza, 1990; Urteaga, 2010; Jahrau; Nassehi, 2012). Siguiendo esta argumentación, la realidad es "una construcción”, “ya que esta diferencia entre autorreferencia y referencia externa no puede existir en el entorno del sistema" (Luhmann, 1996:16-17). Lo que se entiende por realidad es la síntesis de las operaciones internas del sistema y las operaciones externas procedentes de otros sistemas excluyéndose de esta forma una realidad surgida del entorno, por lo que se descarta "una" realidad objetiva. A pesar de un cierto soliptismo de esta tesis, en última instancia el observador define y diferencia lo que es posible entender por realidad.

Habitualmente los ámbitos en que el sistema político -sus representantes los políticos- se mueven, y los roles del público se encentran delimitados y con poca predisposición comunicativa (participativa). Los medios de comunicación de masas asumen una serie de funciones al respecto. En la perspectiva sistémica autorreferencial de Luhmann (2014:328-239) se destacan unas funciones básicas para el mantenimiento del orden y la lealtad al sistema político. En primer lugar tienen la función de garantizar la "pasividad del observar". Con ello la función de observar queda separa la necesidad de presencia, manteniéndose separados los roles individuales de otros más generales según el rol. El resultado es la pasividad del rol del espectador de la política. En segundo lugar, operan de forma "multifuncional" o sea, como forma de ejercer funciones específicas entre las que destaca como principal fabricar "síntomas de voluntad" o voluntades entre los electores. Por último aparecen

3. En la perspectiva de la sociología política Berger y Luckman atribuyen al lenguaje posibilidad de la como una serie de fenómenos interacción social (de forma semejante a Habermas, 1999, 1981) y al lenguaje un papel decisivo para la construcción de la realidad 
también funciones no políticas de tipo económico y empresarial sobre las que no profundizaremos aquí.

Ya adelanta Alfred Schütz que el periodismo desempeña en la actualidad una centralidad indiscutible en la mediación simbólica de la realidad -en este caso política- colectivamente compartida. En situaciones de crisis -como es la situación de desencanto sistémico que afecta a todas las esferas de la vida y el trabajo en el Brasilla información periodística alimenta unos resortes que determinan la construcción de la realidad política (Casero, 2009) 4 . En su propuesta sociológica de "recontextualización" promueve una "doble dinámica" de transformación y conservación en un doble sentido. De una parte, con la difusión de nuevos significados, "derivados de la reintegración de lo atípico" con el objetivo de modificar el conocimiento y así transformar unas definiciones de la realidad social y política. De otra parte, a partir del mantenimiento del orden institucional general de referencia, en el que se pretende la "preservación de la estabilidad del mundo social e impidiendo su posible fractura" y consiguiente su deterioro. Los resultados de esta dinámica son claros: al aparecer en los medios una "sobrerepresentación" de la política y con ello orientar el proceso de formación de opiniones. Sin embargo, su propuesta se limita a un plano individual en la que las operaciones de los sistemas que forma la sociedad no son abordadas. Vale aquí indicar que el grupo multimedia $O$ Globo tiene una cobertura con sus informativos de más de doscientos millones de ciudadanos. En el punto que sigue analizamos las operaciones y las codificaciones de los sistemas jurídico, políticoadministrativo y económico.

\section{Operaciones comunicativas entre los sistemas jurídico y político-administrativo}

El combate a la corrupción ha sido el mensaje central de los sistemas político administrativo y judicial en torno a justificar los procesos contra los expresidentes. A modo informativo, en Brasil 1.833 alcaldes (el 16,5 por ciento) y 11.171 concejales están acusados de crímenes de diferente naturaleza ${ }^{5}$.

Una propuesta conceptual que vincula el fenómeno de la corrupción con la Ciencia política y el Derecho, se fundamenta en la hipótesis de que la corrupción puede ser vista también como un problema de gobernanza. Gobernanza pública significa que los gobiernos deben ser eficaces, no solamente en la capacidad de gestión, sino también con el respeto de las normas y valores imperantes en la democracia.

4. Inspirándose en Alfred Schütz, Peter Berger y Thomas Luckmann, propone la idea de sociofenomenología

5. De este total 99 de alcaldes esta acusados por actos directos de corrupción (ALM Consulting. Mapa da corrupção, 2017). Los más frecuentes son, por orden: finanzas públicas contra la Administración en general, crímenes de responsabilidad y crímenes en la Ley de licitaciones, y crímenes ante finanzas públicas (MPF, Combate a la corrupción, 2017). 
Kooiman (1999) amplia la definición de gobernanza, otorgando al término aspectos en que destaca la actitud humana y la importancia de la existencia de reglas de calidad en los sistemas en relación a la actividad humana.

Las interpretaciones sociológicas adolecen de unas limitaciones teóricas a la hora de explicar la complejidad de las sociedades actuales y los fenómenos que tienen lugar en ellas. La mayoría de sociólogos coincide en que la sociedad está constituida por comunicaciones, siendo la corrupción una de estas. En este consenso aparecen diversas opiniones cuando se mantiene que también está constituida por sistemas, sea en la perspectiva de la Ciencia política (Mayntz; Keohane, Pateman, Lindblom) o en la sociológica (Habermas, Parsons, Bourdieu, Giddens, Luhmann). Las sociedades avanzadas se caracterizan por la pérdida del centro jerárquico ("sociedad policéntrica”) y las limitaciones del poder en base a un único órgano central (no jerarquizada).

Asumimos la premisa sobre la existencia de tres sistemas principales (jurídico, político-administrativo y económico) y también de diversos sistemas parciales o subsistemas. Cada uno de estos realiza funciones diferentes (salud, educación, fuerzas armadas, cuerpos de seguridad, justicia, hacienda pública, autarquías, agencias estatales) que actúan de forma independiente e interdependiente, y cuyo objetivo es reducir la complejidad del sistema global del Estado. Estos subsistemas poseen características autopoiéticas o autorreferentes ${ }^{6}$, lo que significa que son sistemas cerrados (en sus estructuras) aunque abiertos en el plano cognitivo, puesto que están acoplados estructuralmente con el objetivo de permitir el funcionamiento de la totalidad del Estado. La acción político-administrativa del Estado (políticas públicas, leyes, decretos, programas), se manifiesta a través de comunicaciones entre los subsistemas. La teoría de sistemas sociales complejos o autorreferenciales, elaborada por Niklas Luhmann (aquí, 2014; 2004; 1993; 1988; 1988a; 1987) aporta un sofisticado y complejo marco de referencia conceptual.

Los elementos de un sistema social son comunicaciones con capacidad para producir comunicaciones adicionales. En un plano social, un sistema social puede reproducirse a sí mismo siempre que exista una comunicación dinámica. Para observar el fenómeno brasileño sobre los escándalos de corrupción, inicialmente algunos observadores establecieron la diferenciación "silencio/manifestación política". Sobre esta diferenciación inicial es posible establecer la diferenciación a un nivel social del fenómeno de la corrupción como normalidad / excepción. Esta diferenciación asimétrica nos permite pensar otro nivel social en un contexto colectivo y social de democracia deliberativa, como propone Jürgen Habermas (1991).

6. La autorreferencialidad hace referencia a la formación de las estructuras dentro del sistema. Un sistema autorreferencial, es un sistema autopoiético que puede operar cerrado o sometido a la clausura operacional y con capacidad de auto-creación de sus propias estructuras. 


\section{Sistema jurídico}

En la perspectiva sociológica de la teoría de sistemas, el sistema jurídico forma parte de los tres principales sistemas funcionales diferenciados en los que se construye el sistema social, en las que su acoplamiento estructural se mantiene -según Luhmann $(1984 ; 1988)$ - a partir de comunicaciones basadas en códigos binarios. En el transcurso histórico el sistema jurídico ha sido descrito como un sistema aparte del sistema social, vinculado solo en algunos aspectos, y en el que la sociedad formaría tan solo parte de su entorno. Es necesario replantear este erróneo supuesto. El sistema jurídico no solo forma parte de la sociedad, sino que constituye una imagen que refleja el sistema de la sociedad. Luhmann diferencia entre sistema de derecho y sistema jurídico. El sistema de derecho gana su cierre operativo mediante la codificación del binomio justo-no justo, "que no sucede con ningún otro sistema" (Luhmann, 1988:126). Es evidente que en los casos que exponemos y con las pruebas presentadas (aunque circunstanciales) el derecho ha emitido la comunicación de "no-justo". Se crea una paradoja al existir otros casos similares recientes de mandatarios que han sido declarados como "justos", mientras en el caso de Lula ha sido declarado como "injusto" (culpable).

Nuestra hipótesis en este trabajo viene a ser que en la capacidad operativa de autoobservación del derecho, éste ha asumido códigos procedentes del sistema político-administrativo (en Luhmann, poder-no poder), dirigidos a la construcción política de la necesidad de un cambio de partido en el poder, en el que el PT debe aparecer necesariamente como "no poder" por medio de la "actuación criminal" de su líder. La defensa demuestra que Lula, lejos de tener conocimiento o haber participado de cualquier "esquema" de corrupción en la empresa estatal Petrobras, perfeccionó los instrumentos de control por parte de los accionistas para su control y con la creación desde el inicio de su mandato del Ejecutivo, la Controladoria General da União ${ }^{7}$, órgano independiente con la capacidad de fiscalizar, que tenía además la atribución de analizar actos de la Petrobras.

La operación del grupo de trabajo de la "Operación Lava Jato" y del Juicio - con la premisa de que en Brasil existiría un escenario de "corrupción sistémica", similar al que tenía Italia con la "Operación Manos Limpias" (que condujo a la dimisión del Primer Ministro presidente Bettino Craxi). La instrucción judicial ha instaurado hasta una arbitraria "fecha de corte" inserida en las diligencias contra Lula, con lo que se descarta que cualquier referencia antes de 2003, "no viene al caso".

Exponemos de forma sucinta las diligencias redactadas por el Ministerio Público Federal (MPF) sobre el acta de acusación. Estas están dirigidas al Juez Federal de la 13a Vara Federal de la Subsección Judicial de Curitiba: 
Apartado " 2.1 contextualización:

14. Para elegirse al cargo de Presidente de la República LULA formulo una fórmula partidaria que marcó la estructura administrativa federal a partir de aquel momento y que culmino en un esquema criminal dirigido a la corrupción, fraude en las licitaciones y lavado de dinero. LULA recurrió al mencionado cargo por medio de la Coligación formada por el PT, PC do B, PL, PMN, y PCB, y conto en el segundo turno de las elecciones con el apoyo del PPS, PSB y PDT”. (MPF, Acusación, 2016)

En el apartado "1.1.Breve resumen del esquema criminoso", se desarrolla la argumentación:

5. De hecho, LULA decidió en última instancia acerca del montaje y continuidad de una estructura criminal que lo beneficio de diferentes formas: (a) garantizó, durante su mandato Presidencial la gobernabilidad fundamentada en bases criminales, mediante compra de apoyo político; (b) formó en favor de su partido - PARTIDO DOS TRABALHADORES [PT] -, un "colchón" de recursos ilícitos para abastecer futuras campañas electorales, no contexto de una perpetuación criminal en el poder; (c) disponibilizó y se aprovechó del dinero derivado de criminales, propiciando enriquecimiento ilícito. Todas estas ventajas indebidas estuvieron vinculadas al desvío de recursos públicos y al pagamento de propinas a agentes públicos y políticos, gremios de partidos y operadoras de finanzas". (MPF, Acusación, 2016)

Para los abogados en esta línea de actuación Lula tendría que, de alguna forma, en algún momento y a cualquier costo, ser investigado y procesado para el supuesto éxito de la "operación" y del esquema que fue mostrado por sus miembros en el Brasil y en el exterior. Para el gabinete de abogados responsable de la defensa de Lula en el auto dirigido al Juez Federal de la 13 ${ }^{a}$ Vara Federal Criminal de la Sección Judiciaria de Curitiba, reproducimos los siguientes parágrafos ${ }^{8}$ (Ação Penal n. 504651294.2016.4.04.7000):

"El interminable "contexto" narrado en la denuncia es una excrecencia, una anomalía jurídica, pues no vehicula concretamente una acusación contra el EXPRESIDENTE LULA - y, por evidente, no puede ser objeto de valoración de este Juicio" (Teixeira, et.al, 2017:7). 
"De cualquier forma, se trata de narrativa que además de haberse mostrado falsa, revela completo desconocimiento (¿o ceguera deliberada?) de los subscritores de la denuncia respecto (i) las estructuras y de los organigramas del gobierno federal; (ii) de la estructura, del tamaño y del complejo sistema de control - interno y externo - de la Petrobras; y, aún, (iii) de las diversas medidas adoptadas pelo EXPRESIDENTE LULA para establecer en el País un sólido y perfeccionado sistema de combate a la criminalidad, con especial énfasis en el enfrentamiento de la corrupción y del lavado de dinero". (Teixeira, et al., 2017:7-8)

Referente al esquema de desvío de fondos de la empresa Petrobras, el Ministerio Público Federal argumenta:

"6. El avance de la investigación revelo, todavía, un escenario de macro-corrupción más allá de la PETROBRAS, en que la distribución de los altos cargos en la Administración Pública Federal, incluyendo las Directorios de la PETROBRAS, era, por lo menos en muchos casos, un instrumento para la recaudación de propinas, en beneficio de enriquecimiento de agentes públicos, de la perpetuación criminal en el poder y en la compra de apoyo político de gremios con la finalidad de garantizar la fidelidad de estas al gobierno LULA. Las propinas eran arrecaudadas por los detentores de posiciones de prestigio en entidades públicas, de particulares que se relacionaban con tales entidades, directamente o por medio de intermediarios, para ser en seguida distribuidas entre operadores, funcionarios y sus padrinos políticos" (MPF, Acusación, 2016).

El conjunto de operaciones que inducen y permiten el lavado de dinero constituye otra acusación (MPF, 2016:90) fundamentados en los siguientes antecedentes:

"162. a) organización criminal, formada por empresarios de la OAS y de diversas otras contratistas, funcionarios públicos de la PETROBRAS, agentes políticos y operadores financieras; b) cartel, practicado por la asociación de contratistas para defraudar el carácter competitivo de licitaciones públicas de la PETROBRAS y enriquecerse ilícitamente; c) fraude a la licitación, hecha por medio de ajustes oscuros realizados entre concurrentes, con el auxilio de funcionarios públicos; d) corrupción activa y pasiva, siendo algunos actos de esta naturaleza objeto de esta denuncia; e) crímenes contra el orden tributario, pues las contratistas vinculadas en el esquema criminal utilizaron de documentos falsos, numerosas notas fiscales y contratos fraudulentos, para justificar pagamentos sin justificación, reduciendo ilícitamente o reduciendo los impuestos que incidieron en operaciones de esta naturaleza; y f) crímenes contra el sistema financiero nacional, especialmente la operación de institución financiera sin la autorización, la realización de contratos de cambio con informaciones falsas y la evasión de divisas" (Ibid., 2016). 
Para la defensa de Lula, desde el inicio de su gobierno fueron creados y perfeccionado órganos con mayor capacidad para fiscalizar la corrupción, inexistentes hasta entonces en el país. En el informe de la defensa del gabinete de abogados:

"El EX-PRESIDENTE LULA, lejos de tener conocimiento o haber participado de cualquier "esquema" de corrupción en la Petrobras, perfecciono sobremanera el método y el instrumental de control por parte de la accionista controladora (capital votante), a Unión, creando el primer día de su gobierno un órgano independiente e con enorme y eficaz capacidad de fiscalización, la $\mathrm{CGU}^{9}(\ldots)$ incluso con atribución especifica para analizar actos de la Petrobras". (Teixeira, et al., 2017:8)

En el proceso contra Lula, para los abogados de su defensa, desde la fundamentación de la acusación hasta las aparentes pruebas pretenden mostrar una clara culpabilidad sin la existencia de ninguna prueba, sino en interpretaciones subjetivas y proclives a ser interpretadas según los intereses del jurado:

"Definitivamente, esse falacioso "contexto" não passa pelo mais elementar teste de inferências ou mesmo pela - inconstitucional e exótica - teoria "explicacionista" (...) se exibe propia de regimes totalitarios y con remarcado tinte de persecución. HITLER en su primer discurso como Canciller de Alemania defendió exactamente la "elasticidad de los veredictos"'”. (Teixeira, et al.,2017:8-9)

La acusación principal sobre presunto recibimiento de un apartamento (tríplex) se mantiene solo bajo suposiciones extraídas de declaraciones premiadas de aparentes testigos, sin la existencia de pruebas jurídicas o notariales referentes a la aceptación de su propiedad o titularidad:

"Sobre el apartamento en el litoral paulista, denominado tríplex, además de la escasa prueba producida en los autos estar a indicar que el EX-PRESIDENTE LULA jamás tuvo a pose y mucho menos la propiedad de este inmueble - que el compareció una única vez para evaluar (y rechazar) interés en su compra" (Ibid.10).

"Tales imputaciones se basan única y exclusivamente en declaraciones concedidas y en declaraciones premiadas y no poseen cualquier tipo de comprovación real. Incluso no podrían, ya que el EX-PRESIDENTE LULA jamás participo de ningún esquema de corrupcción que venga a ser apurado, ni siquiera tenia conocimiento de su existencia”. (Teixeira, et al., 2017:357)

9. Controladoria Geral da União, Medida Provisional nำ103, de 1/01/2003, posteriormente convertida en Ley $10.683 / 2003$. 
El informe de los abogados concluyen que:

"Además de estos, el EX-PRESIDENTE LULA y su familia jamás tuvieron ni siquiera la pose del inmueble. La pose, como se sabe, constituye elemento imprescindíble para substanciar la acusación referente a la ocultación y/o disimulo de propiedad". (Ibid., 2017:260)

El Ministerio Público Federal también está cometiendo irregularidades con las informaciones obtenidas al reproducirlas de forma acrítica y permitir su difusión con el objetivo de desprestigiar a Lula:

"El MPF abuso, aquí, en la creación de hipótesis acusatorias en busca de resultados políticos propios de lawfare - con el objetivo de molestar o impedir la actuación política de aquellos que es actualmente y sin ningún favor al mayor líder de la oposición del País - e todavía, la creación de un "producto comercial" que actualmente está vendiendo películas, libros, series para TV e incluso conferencias en eventos de congresos de - dígase - de cirugía plástica". (Teixeira, et al., 2017:9)

En este parágrafo el gabinete de abogados hace pública la injerencia que personas ajenas a las investigaciones cuyos nombres no han sido revelados y que han tenido acceso a material confidencial y a filmaciones realizadas por la Policía Federal. Todo este material reservado ha sido destinado para la realización de una película (Película sobre el Lava Jato y un inversionista secreto) (Folha de S. Paulo, febrero 2017). Este hecho constituye un caso grave de injerencia en pruebas y de acceso indebido al material, sin que fuera tomada ninguna medida. La película "A lei é para todos" constituye uno de los productos comerciales que la operación Lava Jato ha producido con el nombre de Lula, utilizando material no autorizado según consta en la Constitución Federal. Por otro lado, el reciente libro de un periodista hace referencia hasta en los pensamientos sobre el proceso del juez Sérgio Moro. El magistrado ha estado presente en la presentación del libro, dando autógrafos y refrendando su contenido. Los derechos del libro han sido vendidos a la productora Netflix para una producción de una serie ${ }^{10}$. Con estos hechos se trata de productos comerciales sin un consentimiento de Lula.

10. La película A lei é para todos, es uno de los productos comerciales que Lava Jato produjo con el nombre de Lula, utilizando material que según la Constitución Federal debería estar protegido por el Estado. 


\section{Sistema político-administrativo}

El sistema político-administrativo -como todo sistema- es un sistema autorreferente (que produce y reproduce por sí mismo los elementos). Está formado por la política (como "un sistema social complejo autónomo bajo limitaciones estructurales propias" (Luhmann, 1993:63) y la administración (hace referencia a la totalidad de las organizaciones que se apoyan en mandatos políticos). En la conjunción de ambos sistemas aparece la corrupción como operación interna y como forma de comunicación. La corrupción y los crímenes de diferente naturaleza contra la Administración pública se han convertido en operaciones para el mantenimiento de la autorreferencialidad del sistema político. En la complejidad de las sociedades actuales, con rápidos y dinámicos cambios, Luhmann (1993:58) cuestiona sobre el poder seguir hablando de un "auténtico control de los problemas" mediante el código ha veces poco reflexivo de "progresista/conservador". Por esta razón definimos el juego político con el código "poder/no poder". En el caso que exponemos, si bien este código sigue siendo útil, aparece una clara diferenciación entre "progresista/conservadora" al aparecer consignas de algunas fuerzas políticas reaccionarias ("vuelta de los militares", "no a los gays y lésbicas", o "los negros son solo obsesos").

El proceso constitutivo democrático de 1988, el sistema político-administrativo brasileño se ha caracterizado por los continuos casos de corrupción de los partidos en el poder. Ya el primer gobierno democrático después de la dictadura el Presidente Color de Mello (1990-1992) fue acusado de "crimen de irresponsabilidad" y su impeachment. Los siguientes gobiernos no se han librado de este tipo de prácticas y acusaciones (Itamar Franco, 1992-1995) (Dossie corrupção). El gobierno de Fernando Henrique Cardoso (1995-2003) estuvo marcado por diecisiete denuncias y 45 casos públicos de corrupción de diverso tipo (O Jornal de todos os Brasis, 7 sep. 2014) suficientes para que fuera iniciado un proceso de impeachment. La mayoría de denuncias fueron presentadas con mayor frecuencia durante el segundo mandato, siendo la mayoría archivadas o desestimadas por los entonces presidentes de la Cámara de los Diputados.

Entre estas denuncias destacan la compra de votos para su reelección, en la que para cambiar la Constitución (se formó un esquema de compra y venta de votos entre los diputados). Privatización irregular y no transparente de la empresa Companhia Vale do Rio Doce. A pesar de la movilización social para frenar este proceso (la empresa estatal fue vendida en una subasta por aproximadamente mil millones de \$ USA, cuando especialistas de mercado estimaron su precio, al menos, en diez mil millones de \$ USA). Con esta operación se trató de un crimen de "lesa-pátria", ya que la empresa generaba beneficios además de ser estratégica para los intereses del país. El escándalo de la empresa Telebrás, a raíz de la privatización del sistema 
de telecomunicaciones. Se trató de un juego de intereses, lobbys, sin transparencia ni debate público, vinculado con escándalos en los que el nombre del presidente estuvo involucrado. El precio que se pagó por esta empresa nacional estuvo incluso subvencionado por el banco público (BNDES) y por el propio gobierno. El gobierno de FHC rechazó una investigación sobre todos los procesos. Las comisiones y transacciones ilegales sirvieron para la financiación de su campaña y para la financiación de otras privatizaciones y empresas vinculadas a parlamentarios del partido gubernamental.

\section{Impeachment de Dilma Rousseff}

El mandato de la Dilma Rousseff (2011-2016) es otra operación de autorreferencia del sistema político. Estuvo marcado por el inicio de la crisis financiera y económica mundial con fuertes repercusiones en el Brasil, lo que obligó a su gobierno a implementar el mayor recorte en el presupuesto de la historia del país (estimado en unos setenta billones de RS (aprox. 25.000 millones de EUR, cambio 2015). Tres operaciones autorreferenciales en el sistema marcaron su gobierno vinculadas directamente con el sistema económico:

- La denuncia en los medios de comunicación sobre la existencia de la "caixa 2" (financiación ilegal de los partidos

- Las denuncias de irregularidades con la financiación de proyectos e inversiones dudosas en la empresa estatal Petrobras (Senado Federal, 2016, 2016a, 2016b, 2016c, 2016d).

- En el debate (12 de mayo 2016) fue aceptada la interpretación del crimen por las denominadas "pedaladas fiscales" como crimen de responsabilidad (61 votos contra 20) con los cual fue apartada de su cargo por 180 días debido a la instauración del proceso de impeachment teniendo su mandado definitivamente cesado el 31 de agosto de 2016.

Durante el mes de marzo de 2016, surgieron manifestaciones populares en todas las ciudades y as regiones del país (con una participación de unos tres millones de personas para reclamar el fin de la corrupción y la dimisión como presidenta. Estas protestas fueron las mayores manifestaciones populares de la historia del país en la que algunos lanzaban consignas como: "intervención militar", "vuelta de los militares" (Oliveira, 2016).

El impeachment, organizado por las fuerzas políticas conservadoras y de derecha fue protagonizado por su Vice-presidente Michel Temer (PMDB) y se decidió el 17 de abril de 2016, en la Cámara de los Diputados (con 367 votos favorables, 137 con- 
trarios, 7 abstenciones y 2 ausencias) de instaurar el proceso de impeachment contra la presidente Dilma para ser debatido en el Senado Federal. Con ello se encerraba un periodo 13 años y cinco meses del PT en el poder. Las presuntas "pedaladas fiscales" (Engelber y Mattos Advogados, 2016) realizadas por su gobierno no fueron en sí una novedad, ya que ya había sido practicada por los gobiernos de otros ejecutivos anteriores. Durante todo el proceso se puso de manifiesto el papel de los medios de comunicación de masas del país en la creación y construcción de una imagen favorable a su destitución (principalmente con los gigantes mediáticos de O Globo, SBT y $B a n d^{11}$ ) y con la participación de la prensa escrita.

\section{La presidencia de Michel Temer}

Michel Temer (PMDB) Vice-presidente en el gobierno de Dilma Rousseff, asumió el cargo de Presidente la República en 2016. Desde el primer día su mandato ha estado marcado por los escándalos de corrupción sobre su gobierno y su persona han sido constantes. Además de citaciones en testigos del Lava Jato, ya antes de asumir la presidencia estaba considerado "ficha suja" (no apto para ser elegible debido a cargos de corrupción) y de tener un proceso de impeachment pendiente en la Cámara de Diputados.

En la operación Lava Jato, Temer fue citado en cuatro ocasiones. Durante denuncia premiada, un ex-senador declaro que o ex-presidente del PMDB era el "padrino" del ex-director de la BR Distribuidora (empresa) y vinculado en un caso de adquisición ilícita de etanol por medio de una subsidiaria de la Petrobras (Ministerio Público Federal, Inquérito, 2017) Temer bloqueó la indicación de otros testimonios ya condenados por la operación. Ya en el año 2014, su nombre fue mencionado por un intercambio de mensajes entre el propietario de la constructora OAS. El entonces presidente retirado de la Cámara de los Deputados denunció de pagamentos a Temer y transferencias económicas a otros miembros de su partido.

En la Cámara tuvo inicio un proceso de impeachment contra Temer, ya iniciado cuando ejercía de vice-presidente por una decisión del Ministro del STF. La comisión especial que analizó el proceso fue formada por los partidos aliados (PSDB, PMDB y $\mathrm{DEM})$. Frente a las graves acusaciones que recaen sobre el presidente Temer (PMDB) con la revelación de denuncia premiada de empresarios solo pueden ser procesado y juzgado en el Supremo Tribunal Federal acepten la denuncia por infracción penal. A finales del 2017, era denunciado por corrupción pasiva. La primera denuncia por sospecha de crimen de corrupción, fue parada por la Cámara de los Diputados al no autorizar para que el STF analizara el caso.

11. Tan solo los tres grupos citado dominan el 70 por ciento de la audiencia. Globo ocupa el 19 lugar en el ranking mundial como conglomerado entre los mass media. 


\section{Conclusiones}

La aplicación del derecho ha recibido y operado comunicaciones en forma de códigos procedentes del sistema político-administrativo y del sistema jurídico. La sustitución del código "legal-ilegal" ha sido sustituido por el "poder-no poder" en el que el "no poder" ha estado dirigido desde el inicio del gobierno del PT a su judialización mediante la presentación de "ser el partido corrupto". Los códigos y las comunicaciones (sentencias) ponen de manifiesto esta práctica de sustitución. La autorreferencialidad del sistema jurídico se ha manifestado en la construcción jurídica de "culpabilidad" de Lula. Las operaciones han estado dirigidas a su ingreso en prisión, al ser el simbolismo jurídico fundamental para este sistema.

En el sistema político-administrativo, el black box ha operado simultáneamente de presentar al PT y sus líderes en los mismos términos, con lo que ha sido inevitable "para el bien del país" su reemplazo. Es necesario resaltar que la corrupción no es un fenómeno nuevo en el país. En un primer momento han sido los economistas y su visión del fenómeno los que se han ocupado del fenómeno. En base a indicadores, se han limitado a expresar el fenómeno argumentos con lo que se justifica desde siempre la corrupción ha acompañado desde la política a los actos de los gobiernos y el mercado (Tabla 1).

También surgen contradicciones y paradojas en los medios de comunicación de masas. El domingo 8 de abril, el Chico Pinheiro (periodista del Journal Nacional) informaba al país sobre la prisión de Lula, declaró: "Realizaran el fetiche. El fetiche de ellos era Lula en la cárcel. No fue de la forma que ellos querían, pero Lula fue. ¿Y ahora? ¿Qué van a hacer ahora? ¿Cómo queda todo? ¿Cuál es el próximo paso? Que Lula tenga calma y sabiduría, inspi.... De inmediato el director general de informativos y periodismo de la red $O$ Globo, Ali Kamel acusó al periodista de imparcialidad con el argumento: "El daño está hecho".

El futuro es incierto y cargado de incertidumbres. Diversas encuestas realizadas en abril (2018) daban a Lula un 31 por ciento de votos y al candidato de extrema derecha Jair Bolsonaro (PSC-RJ) un 15 por ciento de votos en un primer turno. El presidente Michel Temer tiene un grado de rechazo del 64 \% (abril 2017) una aprobación de 7 por ciento (marzo 2018). La Marina Silva se mantiene como tercera en intención de voto con un $10 \%$. En un eventual segundo turno, con la participación de Lula, se mantiene estas tendencias ${ }^{12}$. En las entrevistas de intención de voto para las próximas elecciones generales (Datafolha, enero 2018) en una primera vuelta Lula obtendría el 34 por ciento, seguido por Jair Bolsonaro con un 15 por ciento y otros divididos con un 10 y 9 respectivamente. En una eventual segunda vuelta, Lula

12. Encuesta Datafolha realizada entre los días 26 y 27 de abril de 2017, con 2.781 entrevistados. 
obtendría un 51\% seguido de Bolsonaro con un $33 \%$, Este candidato está apoyado por la Iglesias Evangélicas y el partido Brasil Libre (su campaña se caracteriza por el odio al "rojo" negro, gay, inmigrantes y mujeres).

La segunda vuelta de las elecciones presidenciales que tuvieron lugar el pasado 28 de octubre de 2018, han conformado los pronósticos -y presagios- con el triunfo del candidato de extrema derecha Jair Bolsonaro con 55.13 por ciento de los votos, quedando el candidato Fernando Haddad con 44.87 por ciento. Destaca en estas elecciones el elevado número de votos nulos y en blanco que suman un total de 27.5 por ciento, y que representa a casi 30 millones de electores. Una lectura de este resultado es que la suma de votos blancos, nulos y de Haddad ofrece un resultado de casi 80 millones de votantes que o bien no aceptan, o desconfían, del presidente electo Jair Bolsonaro.

Entretanto el sistema de justicia en Brasil sigue con las incertidumbres politizadas. Mientras que el Ministro de Justicia tomaba la decisión de liberar a 169.000 presos -entre los que se encuentra Lula- al día siguiente, el 19 de diciembre el Supremo tribunal Federal revocaba esta decisión.

Tabla 1

Las racionalidades sistémicas entre política, administración y justicia

\begin{tabular}{l|l|l|l}
\hline & Racionalidad política & Racionalidad administrativa & Racionalidad jurídica \\
\hline Perspectiva formal & $\begin{array}{l}\text { Coaliciones } \\
\text { Cambios en los procesos } \\
\text { Formación de opinión }\end{array}$ & $\begin{array}{l}\text { Legalidad/legimitidad } \\
\text { Eficacia efectividad } \\
\text { Creación de decisiones } \\
\text { vinculantes }\end{array}$ & $\begin{array}{l}\text { Impartir justicia } \\
\text { Rapidez en las sentencias }\end{array}$ \\
\hline $\begin{array}{l}\text { Perspectiva } \\
\text { autorreferencial }\end{array}$ & $\begin{array}{l}\text { "Poder / no-poder" } \\
\text { Sistema autónomo } \\
\text { limitaciones estructurales } \\
\text { propias }\end{array}$ & $\begin{array}{l}\text { "Racional/no-racional" } \\
\text { Se apoya en mandatos } \\
\text { políticos vinculantes }\end{array}$ & $\begin{array}{l}\text { Gana cierre operativo } \\
\text { mediante justicia/no- } \\
\text { justicia } \\
\text { Autorreferencialidad en } \\
\text { las leyes }\end{array}$ \\
\hline $\begin{array}{l}\text { Factores críticos en el } \\
\text { caso expuesto }\end{array}$ & $\begin{array}{l}\text { Injerencia con los códigos } \\
\text { al sistema jurídico } \\
\text { Operaciones } \\
\text { autorreferenciales } \\
\text { dirigidas a la construcción } \\
\text { política del “partido } \\
\text { corrupto" }\end{array}$ & $\begin{array}{l}\text { Códigos procedentes del } \\
\text { sistema político } \\
\text { Creación de decisiones } \\
\text { vinculantes apoyadas en el } \\
\text { mandato político. } \\
\text { Decisiones orientadas al } \\
\text { desprestigio público del PT. }\end{array}$ & $\begin{array}{l}\text { Las consecuencias en } \\
\text { la sociedad se derivan } \\
\text { según lo que sucede en el } \\
\text { sistema de derecho } \\
\text { Juez Sérgio Moro: "hicimos } \\
\text { lo que el pueblo esperaba" }\end{array}$ \\
\hline \hline
\end{tabular}


Con el ingreso en prisión del ex presidente Luiz Ignacio Lula da Silva el 8 de abril 2018 se ha abierto un periodo marcado por la inseguridad y el riesgo político en un escenario influenciado por la emergencia de nuevos populismos de extrema derecha. El objetivo final de todos estos complejos procesos, ha sido evitar una posible victoria del Partido dos Trabalhadores (PT) en las elecciones de 2018, las intenciones de voto superaban (en abril de 2018) con el 31 por ciento de votos, superando al resto de candidatos y candidatas.

La corrupción es un fenómeno sistémico-estructural en el país, por lo que de poco sirven las propuestas de políticas públicas y de control de los partidos políticos, si estas políticas tan solo focalizan y combaten la corrupción es sus aspectos visibles con el derecho penal y en forma de medidas represivas.

\section{Bibliografía}

AGUIAR, Vilma (2015) "Um balanço das políticas do governo Lula para a educação superior: continuidade e ruptura”, Revista de Sociología e Política, 24(57):113126. Doi 10.1590/1678-987316245708

ALM Consulting (2017) Mapa da corrupção. Disponible en web: http://www. amlreputacional.com.br/aml-na-midia/o-mapa-da-corrupcao (consulta: 13 de noviembre 2017)

ARAGAO, Eugênio José Guilherme de (2017) “O Risco dos Castelos Teóricos do Ministério Público em Investigações Complexas”, p. 51-59, en: Zanin Martins, Cristiano; Teixeira Zanin Martins, Valeska y Valim, Rafael (coord.), O Caso Lula: A Luta Pela Afirmação dos Direitos Fundamentais no Brasil. São Paulo: Contracorrente.

BAECKER, Dirck (1999) Organisation als System. Frankfurt: Suhrkamp.

BASTOS, Pedro Paulo (2017) "Ascensão e crise do governo Dilma Rousseff e o golpe de 2016: Poder estrutural, Contradição e Ideologia”, Revista Economia Contemporánea, 1-63.

BERGER, Peter; Luckmann Thomas (1968) La construcción social de la realidad. Buenos Aires: Amorrortu.

BÖHNISCH, Lothar (2006) Politische Soziologie. Opladen: Budrich.

BÖRSEL, Tanja; Risse, Thomas (2010) “Governance without a State: Can It Work?”, Regulation and Governance, (4): 113-134.

CASERO, Andreu (2009) La construcción mediática de las crisis políticas. Madrid: Fragua.

DE PAULA, Marilene (2011) "Nunca antes na história deste país”...? Um balanço das políticas do governo Lula. Río de Janeiro: Heinrich Böll Stinftung. 
FARIAS, Ignacio; Ossandón, José (2011) Observando Sistemas. Nuevas Aportaciones y Usos de la Teoría de Niklas Luhmann. Santiago: RIL editores.

FORTUNA, Danielle et al. (2012) "O imaginário político e a doença do presidente Lula na mídia: cobertura jornalística das revistas Veja, Carta Capital, Isto É e Época sobre a doença do ex-presidente Lula”, Temática, 8(6). Disponible en web: http://www.periodicos.ufpb.br/index.php/tematica/article/view/23688 (consulta: 3 de marzo 2018)

HABERMAS, Jürgen (1992) Historia y crítica de la opinión pública: La transformación estructural de la vida pública. Barcelona: Editorial Gustavo Gili.

IZUZQUIZA, Ignacio (1990) La sociedad sin hombres. Barcelona: Anthropos

JAHRAU, Oliver; Nassehi, Armin (Hrsg.) (2012) Luhmann Handbuch. StuttgartWeimar: J.B. Metzler.

JAPP, Klaus Peter (2008) Actores políticos. Estudios Sociológicos de El Colegio de México, . XXVI(76), Disponible en web:

http://estudiossociologicos.colmex.mx/index.php/es/article/view/274/274 (consulta: 22 octubre 2017)

JAPP, Klaus Peter; Kusche, Isabel (2004) Die Kommunikation des politischen Systems: Zur Differenz von Herstellung und Darstellung im politischen System. Zeitschrift für Soziologie, 33(6):511-531.

KOOIMAN, Jan (2008) "Exploring the Concept of Governability, Jornal of Comparative", Policy Analysis: Research und Practice, 10(2): 171-190. https://doi. org/10.1080/13876980802028107

LEYDERSDORFF, Loet (2015) Una teoria sociológica dela comunicación. México DF: Universidad Iberoamericana.

LUHMANN, Niklas (2014) Sociologia política. Madrid: Trotta.

LUHMANN, Niklas (1987) Soziale Systeme. Grundriß einer allgemeinen Theorie. Frankfurt a.M., Suhrkamp.

LUHMANN, Niklas (1988) Ökologische Kommunikation. Opladen, Westdeutscherverlag.

LUHMANN, Niklas (1988a) "Politische Steuerung: Ein Diskussionsbeitrag", en: Hartwich, Hans-Herman (Hg.), Macht und Ohnmacht politische Institutionen. Opladen, Westdeutscher Verlag.

LUHMANN, Niklas (1984) "Soziologie des politischen Systems", en Soziologische Aufklärung, Wiesbaden: Springer, pp. 154-177.

LUHMANN, Niklas (2004) Die Realität der Massenmedien. Wiesbaden: VS Verlag für Sozialwissenschaften. 
MATTOS Engelberg (2016) Advogados. Excelentíssimo Senhor Senador Raimundo Lira, Presidente da Comissão Especial do Impeachment. Denúncia por Crime de Responsabilidade $\mathrm{n}^{\circ} 01 / 2016$.

MAYNTZ, Renate (2009) Über Governance. Institutionen und Prozesse politischer Regelung. Schriften aus dem Max-Planck-Institut für Gesellschaftsforschung, Bd. 62. Frankfurt a.M.: Campus.

MAYNTZ, Renate (2005) “GovernanceTheoriealsfortentwickelteSteuerungstheorie?”, en F.G. Schuppert (Hrsg.), Governance-Forschung. Baden-Baden, pp. 11-20.

NASSEHI, Armin (2008) "Rethinking functionalism. Zur Empiriefähigkeit systemtheoretische Soziologie", en Herbert Kalhott et.al. Theoretische Empirie. Die Relevanz qualitativer Forschung. Frankfurt a.M.: Suhrkamp, pp. 79-106.

OLIVEIRA, Hebe Maria (2016) A construção do impeachment de Dilma Rousseff pela mídia brasileira comercial na cobertura das manifestações de rua. Encontro Regional Sul de História da Midia, 15-16 junho, Paraná.

PARTIDO DOS TRABALHADORES. A Carta ao Povo Brasileiro (2002) Disponible en web: http://www1.folha.uol.com.br/folha/brasil/ult96u33908.shtml (consulta: 5 de marzo 2018)

RATTINGEN, Hans (2009) Einführung in die politische Soziologie. Oldenburg: De Gruyter.

SADER, Emir. (org.) (2013) Lula y Dilma. Diez años de gobiernos posneoliberales en Brasil. IAEN. Instituto de Altos Estudios nacionales del Ecuador. Quito: Boitempo editorial/ Flacso Brasil. Disponible en web: https://www.traficantes.net/sites/ default/files/pdfs/tds_practicas3_lula_y_dilma_web.pdf (consulta: 1 de abril de 2018)

SCHUTZ, Alfred. [1932] (1972) Fenomenologia del mundo social. Buenos Aires: Paídos.

TEIXEIRA, Martins Advogados (2016) Excelentíssimo Senhor Doutor Juiz Federal da $13^{a}$ Vara Federal Criminal da Seção Judiciária de Curitiba/PR. Ação Penal n ${ }^{\circ}$ 5046512-94.2016.4.04.7000. Disponible en web: http://www.lula.com.br/sites/ default/files/anexos/alegacoes_finais.pdf (consulta: 6 marzo de 2018)

TEIXEIRA, Martins Advogados (2016) Excelentíssimo Senhor Doutor Desembargador Federal João Pedro Gebran Neto, do Egrégio Tribunal Regional Federal da $4^{a}$ Região. Razões de Apelação. Disponible en web: http://politica.estadao.com.br/ blogs/fausto-macedo/wp-content/uploads/sites/41/2017/12/10_Razapela1.pdf (consulta: 17 marzo de 2018)

URTEAGA, Eguzki (2010) "La teoria de sistemas de Niklas Luhmann", Contrastes. Revista Interdisciplinar de Filosofía, 15(2-1): 301-317. 
VILAJOANA, Josep M. (2010) "La construcción social de la realidad jurídica", Prismasocial. Revista de Ciencias Sociales, n. 4.

WILLKE, Helmut (2016) “Formas de autoorientación de la Sociedad". Revista Mad, (34): 1-35.

WILLKE, Helmut (1997) Supervisión des Staates. Frankfurt a.M.: Suhrkamp.

ZAHLUHT, Pedro Paulo (2017) "Ascensão e queda do governo Dilma e o golpe de 2016: Poder estrutural, Contradição e Ideologia", Revista de Economia Contemporânea, número especial, 1-63.

\section{Documentos institucionales}

Brasil

Ministério Público Federal. Procuradoria da República do Paraná. Força-Tarefa Lava Jato. Disponible en web: http://www.mpf.mp.br/pr/sala-de-imprensa/docs/ DENUNCIALULA.pdf (consulta: 22 de enero 2018)

Poder Judiciário Justiça Federal. Seção Judiciária do Paraná 13a Vara Federal de Curitiba. Disponible en web: https://www.conjur.com.br/dl/sentenca-condenalula-triplex.pdf (consulta: 27 de enero 2018)

Poder Judiciário Justiça Federal. Seção Judiciária do Paraná 13a Vara Federal de Curitiba. Sentença. Disponible en web: http://noticias.r7.com/blogs/helciozolini/files/2017/07/Senten\%C3\%A7a-Lula1.pdf (consulta: 12 de marzo 2018)

Poder Judiciário Justiça Federal. Excelentíssimo Senhor Doutor Juiz Federal da 13a Vara Federal Criminal da Seção Judiciária de Curitiba/PR. Ação Penal no 504651294.2016.4.04.7000. De São Paulo (SP) para Curitiba (PR), 20 de junho de 2017. (consulta: 22 octubre 2017)

Senado Federal. 2016. Secretaria Geral da Mesa. Sentença. Ministro Ricardo Lewandowski. Presidente do Supremo Tribunal Federal e do processo de Impeachemt. Disponible en web: http://legis.senado.leg.br/legislacao/ ListaTextoSigen .action? norma $=562339 \&$ \&id $=16426939 \&$ \&idBinario $=16429344 \&$ mime=application/rtf (consulta: 17 octubre 2017)

Senado Federal. 2016a. Roteiro para a Sessão do Julgamento. Disponible en web: https://www12.senado.leg.br/noticias/arquivos/2016/08/17/veja-aqui-o-roteirodefinido-para-a-sessao-de-julgamento (consulta: 17 octubre 2017)

Senado Federal. 2016b. Parecer n. 726, da Comissão Especial do Impeachment. Gabinete do Senador Antonio Anastasia. Brasília 6 mayo, 2016.

2016c. Da Comissão Especial do Impeachment. Brasília.

2016d. Excelentissimo Senhor Ministro Ricardo Lewandoswi presidente do Supremo Tribunal Federal e o Senado Federal. Resposta à Acusação. 1 junio 2016. 
2017. Ministério Público Federal. Excelentíssimo Senhor Ministro Edson Fachin. No. 4.483/DF. Inquérito. Brasília.

\section{Periódicos}

El País, 2009. 10 noviembre.

Folha de S. Paulo. 2017. Disponible en web: http://www1.folha.uol.com.br/ ilustrada/2017/02/1857844-filmeda-lava-jato-custa-r-15-mi-teminvestidorsecreto-e-estreia-em-julho.shtml (consulta: 6 febrero de 2018)

Haaretz, 2010. 12 marzo.

Le Monde, 2009. 24 diciembre.

Vermelho. 2017. Dalmo Dallari, Educação para a cidadania. Disponible en web: http://vermelho.org.br/noticia/305695-1 (consulta: 26 noviembre de 2017)

O Jornal de todos os Brasis, 2014. Disponible en web: https://jornalggn.com.br/blog/ iv-avatar/45-escandalos-de-fhc (consulta: 9 febrero de 2018) 